\title{
Fuzzy Case-Based Reasoning in Product Style Acquisition Incorporating Valence-Arousal-Based Emotional Cellular Model
}

\author{
Fuqian Shi, ${ }^{1}$ Jiang $X u{ }^{2}$ and Shouqian Sun ${ }^{3}$ \\ ${ }^{1}$ Department of Information and Engineering, Wenzhou Medical College, Wenzhou 325035, China \\ ${ }^{2}$ School of Mechanical Engineering, Southeast University, Nanjing 211189, China \\ ${ }^{3}$ Modern Industrial Design Institute, Zhejiang University, Hangzhou 310027, China \\ Correspondence should be addressed to Fuqian Shi, sfq@wzmc.edu.cn
}

Received 9 October 2011; Accepted 28 December 2011

Academic Editor: Chong Lin

Copyright (c) 2012 Fuqian Shi et al. This is an open access article distributed under the Creative Commons Attribution License, which permits unrestricted use, distribution, and reproduction in any medium, provided the original work is properly cited.

\begin{abstract}
Emotional cellular (EC), proposed in our previous works, is a kind of semantic cell that contains kernel and shell and the kernel is formalized by a triple- $L=\langle P, d, \delta\rangle$, where $P$ denotes a typical set of positive examples relative to word- $L, d$ is a pseudodistance measure on emotional twodimensional space: valence-arousal, and $\delta$ is a probability density function on positive real number field. The basic idea of EC model is to assume that the neighborhood radius of each semantic concept is uncertain, and this uncertainty will be measured by one-dimensional density function $\delta$. In this paper, product form features were evaluated by using ECs and to establish the product style database, fuzzy case based reasoning (FCBR) model under a defined similarity measurement based on fuzzy nearest neighbors (FNN) incorporating EC was applied to extract product styles. A mathematical formalized inference system for product style was also proposed, and it also includes uncertainty measurement tool emotional cellular. A case study of style acquisition of mobile phones illustrated the effectiveness of the proposed methodology.
\end{abstract}

\section{Introduction}

Natural language (NL) is used to describe the real world while NL is full of fuzziness and ambiguity, humans will create a vague concept on each object by using NL, whereas researching on implicit emotion of product style, NL are often unable to adapt to the quantitative calculation and qualitative analysis. Customer's emotional evaluation is hided in NL that needs to be extracted and decomposed into words and adjectives, but separating these terms under such a simple way cannot fully express the human's visual perception of product. In previous studies, it implicated that human emotion can be measured by two-dimensional value, called valence and arousal, where valence denotes the degree of exciting and calm and arousal denotes the degree of positive and negative $[1,2]$, which made important progress 
on presentation of human's emotion. Further research was appeared in medical fields, a large number of medical experiments indicated that two brain-specific chemosensory domains, amygdala and the orbital-frontal cortex, were linked to valence-arousal [3-5]. However, can different words and different degrees of emotional stimuli be corresponded to certainty domains of these two brain chemosensory signal characteristics? This is a very important basis related to the underlying data acquisition and the scientific foundation of product style description model. PA Lewis observed specific brain areas, including the orbital frontal cortex, anterior cingulate, insula, amygdala, brain stem, pons, and striatum, and other parts of the active peak by magnetic resonance imaging (MRI) technique under different emotions (affective words) and varying degrees of stimulation, it indicated that the orbital frontal cortex is highly correlated with arousal, and the amygdale was highly correlated with valence, this work founded a relationship between low-level physiological signals and highlevel semantic concepts [6].

Emotional cellular (EC), introduced by our previous works, was developed based on Labe semantic (LS) and prototype theory (PT) $[7,8]$, the theoretical aspect LS is defined by set theory that demonstrated the semantic representation under final measure of the accuracy of the description, which is mainly based on logical and accurate measure of the quality function and its synthesizing manipulation. However, in practical examples, the distribution status of mass function was much more difficult to learn that required a priori knowledge and statistical methods. Although the LS theory was reasonable by using emotional vocabulary to generate expression which provided a theoretical basis for EC model under probability aspect, but in the semantic space, the LS theory needs to conduce a formal definition of the expression, and the ultimate goal is to obtain independent semantic concept that takes advantage of this concept of semantic content and to determine the fuzzy boundaries inherent in the product hidden emotional elements. PT established the conceptual model for semantic extraction through a neighborhood-based calculation method and also produced a rule-based reasoning model. The basic idea of PT is to extend the concept to certain but unknowable boundaries of a random set- $P_{i}$, which denotes a representation of the concept that belong to classic set. $N_{L_{i}}^{\varepsilon}$ is a neighborhood of concept semantic. So that About $P_{i}$ can describe the uncertainty of concepts. So, it is reasonable that emotional cellular based on valence-vrousal was used to extract implicit emotion of products, and also appears more stable performance especially for uncertainty and inexactly representation of product implicit emotion images [9]. In industrial design (ID) field, the stability appearance of implicit emotion is an important characteristic of the formation of products style, and the product style features always focus on the emotional evaluation on product forms; so, this paper will use the EC model to extract product style employing fuzzy case-based reasoning (FCBR) technologies.

case-based Reasoning (CBR) refers to use of existing experience under a suitable similarity definition to find a solution to the problem, or revise the past cases while making decisions on new case. The advantage of CBR is that system does not need to enter the entire cases in advance, but simply to rely on the reasoning process comparing with current problems and past cases that have more application on text inference and pattern recognition fields [10-12]. The basic idea of this paper is to map each emotional cellular of valence-arousal space into a "case" and the kernel of emotional cellular represents the central of case, that is, the typical value of case. The density function of emotional cellular was defined to the similarity between cases.

While in actual process, CBR method must be combined with fuzzy sets that will take more effective for feature extraction of product style due to the uncertainty on knowledge representation of the products style, form features, attributes description, and the definition of 
similarity measurement; so fuzzy case-based reasoning (FCBR) was introduced subsequently [13-17].

In the 1980s, Schank [18] and his students firstly introduced CBR further improved by Janet Kolodner's and Michael Lebowitz's who developed two software systems called CYRUS and IPP $[19,20]$. Case retrieval is an important direction in CBR research while Hüllermeier et al. [21] used generalization of the similarity measure to improve the efficiency of case retrieval, and fuzzy sets make it even more powerful for CBR. Wu et al. [22] analyzed the application of fuzzy set theory to CBR process of various issues and gave many solutions while other scholars committed to the integration of CBR in fuzzy clustering and fuzzy reasoning to improve the performance of the system.

CBR emphasizes the connection between cases and makes it more feasible for researching the formation of product style. In conceptual design (CD) fields, Cheng et al. [23] used 100 products to establish a FCBR reference model under a given similarity metric method in creative product design system. In summary, CBR is the process of solving new problems based on the solutions of similar past problems, it has its unique advantages on the field of ID; the EC model introduced in CBR will improve the ability of knowledge representation according to the manner of human thinking, and overcome the weakness that CBR's original case presentation always falls into a single logical system and only suitable to clearly defined issues. In EC-based CBR inference process, knowledge base is established easier and more suitable to product style feature extraction [24-26].

\section{Four Steps of Case-Based Reasoning}

Case-based reasoning has been formalized as a four-step process [27-30].

(1) Retrieve: retrieve cases from memory, that are, relevant to solving a target problem. A case consists of a problem that how the solution was derived.

(2) Reuse: the solution from the previous case was mapped to the target problem for that it need to involve adjust the previous solution to fit the new situation.

(3) Revise: test new solution, if necessary; revise it after having mapped the previous solution to the target situation.

(4) Retain: store the resulting experience as a new case in memory after the solution has been successfully adjusted to the target problem (Shown as Figure 1).

\section{Similarity under Fuzzy Nearest Neighbor for Product Style Extraction}

Fuzzy logic can deal with the expression of fuzzy language better, such as, "very good," "good," "bad," and "very bad." Combining with fuzzy sets to CBR process, a fuzzy preference function was defined to calculate the similarity between the corresponding properties for target problems. The result of fuzzy preference function is a vector, called the fuzzy preference vector (FPV) while the vector contains the fuzzy preference value for each attribute; extracting fuzzy preference function allows comparing some particular characteristic under completely different scales. 


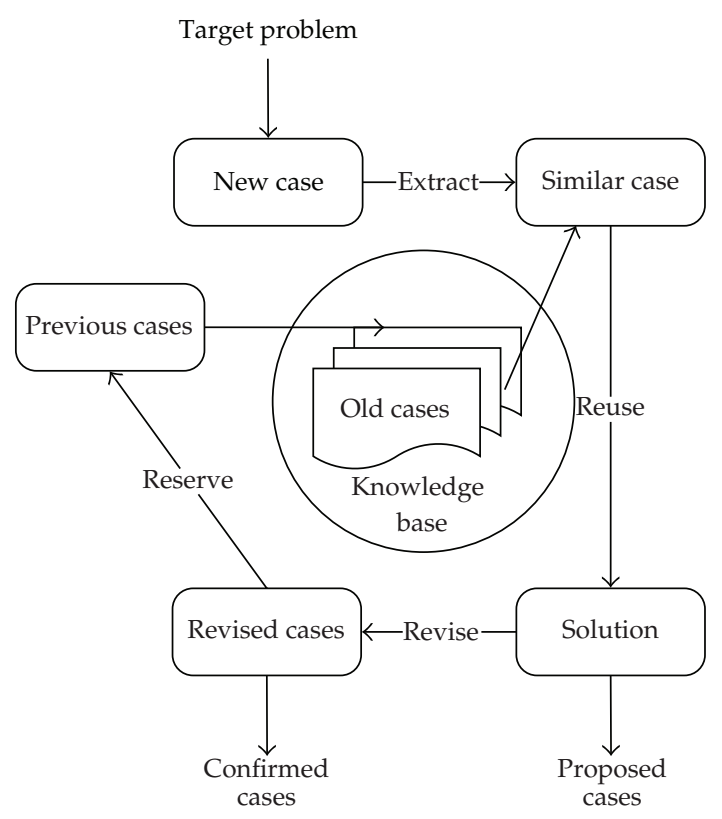

Figure 1: Process of general case-based reasoning.

Nearest-neighbor (NN) technology was used to calculate the similarity between cases, comparing the relative properties for each new case and previous cases under given case weights, the total weight (weighted sum) was calculated and applied to sort the candidate cases. Most of the CBR systems use this method, and the degree of similarity may often be normalized into a number between 0 and 1 , where 0 denotes completely different, 1 denotes exactly same, or as a percentage while $100 \%$ is representative of exactly same. This theory was first introduced by Fix and Hodge [31] and subsequently developed by Glass et al. [32] and Devijver and Kittler [33] and Wilson [34] that made some progress in data mining and image recognition. Kuncheva [35] and Liaw et al. [36] proposed a precise KNN algorithm using orthogonal search tree method to improve the accuracy of pattern recognition.

In the investigation stage, product style features need to be described more closely to natural language. In previous studies, the usual practice was to use linguistic variables $(\mathrm{LV})$; LV is simplify of natural language and also combined fuzzy set theory. Table 1 shows the typical linguistic variable and its scale. However, the membership functions of LV is not linear, the specific value will come from the prior analysis by experts.

Fuzzy nearest neighbor (FNN), first proposed by Keller et al. [37] in 1985, is mainly to describe cases using fuzzy set, its advantage is that system can capture more cases whose fuzzy memberships are bigger than a given threshold, and provide support information for decision making to reduce the errors. A typical FNN algorithm is shown in Algorithm 1 and Figure 2.

While properties of each case may represent different importance degrees, we must set each weight relative to a property in order to find the most similar case more effectively and accurately. For NN algorithm technology, the distribution of weights will help us to acquisition "good" case. However, value selection for weight of property is very difficult, traditionally, weights are defined by experts according to their expertise or experience in practice. 
Table 1: Linguistic variable and its scale.

\begin{tabular}{|c|c|c|c|c|c|c|c|c|}
\hline Scale & 1 & 2 & 3 & 4 & 5 & 6 & 7 & 8 \\
\hline Variables & 2 & 3 & 5 & 5 & 6 & 7 & 9 & 11 \\
\hline None-N, N & & & & & & & & $\mathrm{Y}$ \\
\hline Very low, VL & & & $\mathrm{Y}$ & & $\mathrm{Y}$ & $\mathrm{Y}$ & $\mathrm{Y}$ & $\mathrm{Y}$ \\
\hline Low-very low, V-VL & & & & & & & $\mathrm{Y}$ & \\
\hline Low, L & $\mathrm{Y}$ & Y & Y & Y & $\mathrm{Y}$ & Y & Y & $\mathrm{Y}$ \\
\hline Fairy low, FL & & & & Y & $\mathrm{Y}$ & & Y & $\mathrm{Y}$ \\
\hline Mol low, ML & & & & & & Y & & $\mathrm{Y}$ \\
\hline Medium, M & & $\mathrm{Y}$ & $\mathrm{Y}$ & $\mathrm{Y}$ & & Y & Y & $\mathrm{Y}$ \\
\hline Mol high, MH & & & & & & $\mathrm{Y}$ & & $\mathrm{Y}$ \\
\hline Fairy high, FH & & & & $\mathrm{Y}$ & $\mathrm{Y}$ & & & Y \\
\hline High, $\mathrm{H}$ & $\mathrm{Y}$ & $\mathrm{Y}$ & $\mathrm{Y}$ & $\mathrm{Y}$ & Y & Y & Y & Y \\
\hline High-very high, $\mathrm{H}-\mathrm{VH}$ & & & & & & & Y & \\
\hline Very high, VH & & & $\mathrm{Y}$ & & Y & Y & Y & Y \\
\hline Excellent, E & & & & & & & & $\mathrm{Y}$ \\
\hline
\end{tabular}

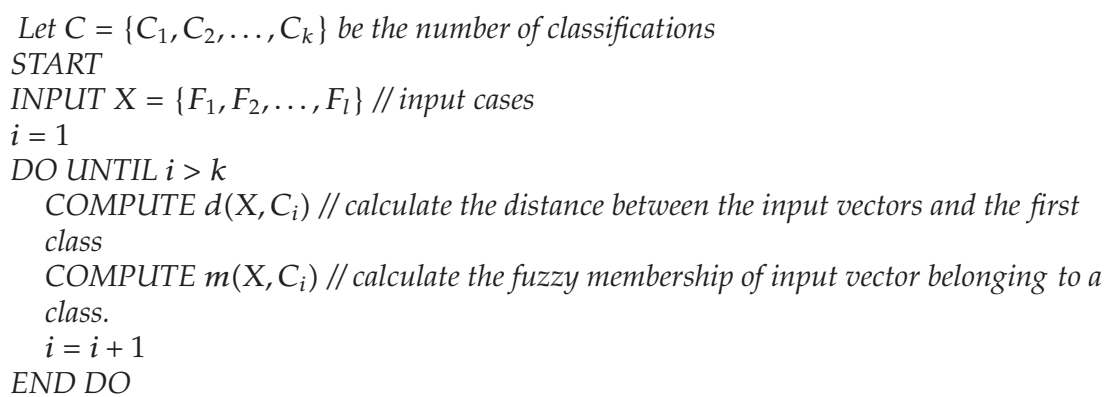

Algorithm 1

Combining with FNN and CBR by using discrete scale-LV cannot be more exact to describe product style, so the EC model needs to be used in this reasoning system. By the above discussion and analysis, the similarity formula for cases is also redefined.

\section{Product Style Acquisition by FCBR Employing EC}

\subsection{Emotional Cellular Theory}

\subsubsection{Definitions on Kernel of Emotional Cellular}

In a previous experimental study [1], we got the distribution of each point set for each emotional vocabulary in valence-arousal space. The analysis revealed that the distribution of different emotions vocabulary in valence-arousal space is not the same and can be divided into three categories: single point type (indicating that the emotional evaluation has a strong consistency), planar type (that poor consistency of the emotional evaluation), and circular type (for the emotional evaluation under a medium consistency). So the spatial distribution 


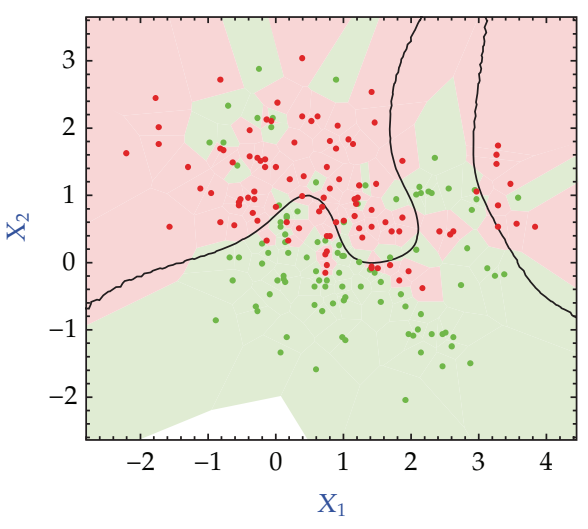

(a)

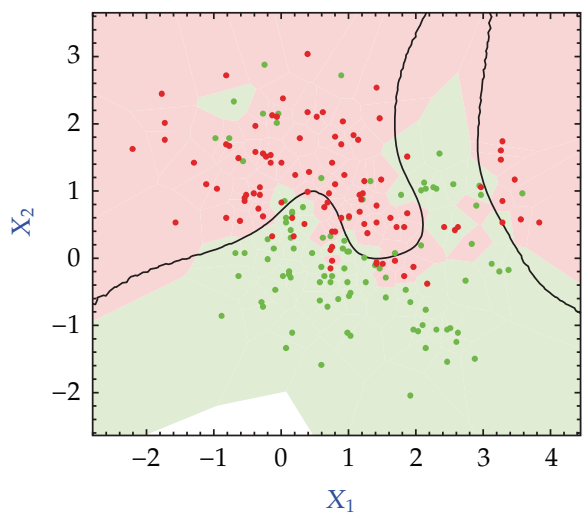

(b)

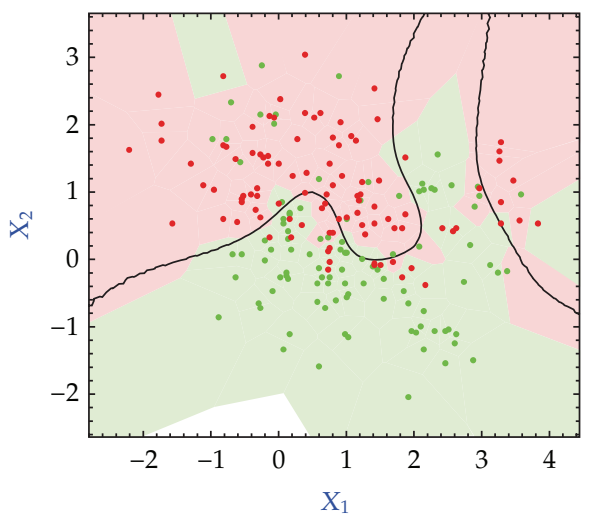

(c)

Figure 2: K-fuzzy nearest neighbor (Keller et al, 1985) and stimuli (by Ian McLeod). K-fuzzy nearest neighbor under $K=3,7,9$.

and its different topological forms of point set need to be redefined respectively. For this situation, The emotional cellular model is actually a very special semantics cell model that is defined on the domain of two-dimensional valence-arousal emotional space which contains two parts: semantics kernel and semantic shell. Semantic kernel is a typical values in valencearousal space, it means that all cells belonging to the emotional element of a typical valencearousal value; emotional shell covers the areas of emotional vocabulary boundary, it is uncertain in nature, which uses a density function to represent the distance uncertainty. The kernel is a classic set that takes many forms such as single-point set and a collection of circular; and its shell also is under a variety of forms of density function, such as single Gaussian model (SGM) and Gaussian mixed model (GMM). This paper will develop SGM and GMM in EC model based on Olvi Mangasarian and Wang's works [38, 39]. Definitions were given as follows.

Definition 4.1. For $\forall P \in \Omega, P$ is a point that belongs to the universe $\Omega=\left\{\left(E_{1}, E_{2}, \ldots, E_{n}\right)\right.$ : $E_{i} \in P$. Particularly, $P=$ (valence, arousal) in valence-arousal emotional space: $P=(v, a)$, $\Omega=\{(v, a) \mid v, a \in R\}$. 
Definition 4.2. Distance $d=\|\cdot\|$ in valence-arousal emotional space, satisfied by the condition as follows:

$$
\begin{gathered}
d(P, P)=\|P\|=\sqrt{\left(v^{2}+a^{2}\right)} \\
d(P \pm Q)=\|P \pm Q\|=\sqrt{\left(\left(v_{P} \pm v_{Q}\right)^{2}+\left(a_{P} \pm a_{Q}\right)^{2}\right)}, \quad \forall P, Q \in \Omega \\
\forall \alpha, \beta \in R, \quad P, Q \in \Omega, \\
d(\alpha P \pm \beta Q)=\|\alpha P \pm \beta Q\|=\sqrt{\left(\alpha v_{P} \pm \beta v_{Q}\right)^{2}+\left(\alpha a_{P} \pm \beta a_{Q}\right)^{2}} .
\end{gathered}
$$

Furthermore, we have that

$$
d(\alpha P+\beta Q) \leq|\alpha| \cdot d(P)+|\beta| \cdot d(Q)
$$

Definition 4.3. for $\forall P \in \Omega$, there is a neighborhood $N_{P}^{\varepsilon}$ :

$$
N_{P}^{\varepsilon}=\{X \mid\|P-X\|<\varepsilon, X \in \Omega\} .
$$

Definition 4.4. Single-point kernel is defined by

$$
\left\{P_{K}\right\}, P_{K}=\frac{1}{\|K\|} \sum_{P_{i} \in K} P_{i} \rho\left(P_{i}\right)
$$

where $K$ is KASNEI adjectives set and $\rho\left(P_{i}\right)$ is the density of $P_{i}$.

Definition 4.5. Circular kernel is defined by $\left\{P_{j} \mid P_{j} \in N_{P_{K}}^{\varepsilon}\right\}$, where $P_{K}=$ (1/ $\left.\left\|K^{\prime}\right\|\right) \sum_{P_{i} \in K^{\prime}} P_{i} \rho(P i), K^{\prime} \subset K$ and $K^{\prime}=\left\{P_{i} \mid \rho\left(P_{j}\right) \leq \rho_{T}\right\}, \rho_{T}$ is a given const to limit the scale of kernel.

Definition 4.6. Flat kernel is defined by $\left\{\bigcup_{i} P_{K}\right\}$, where $P_{K}$ is subject to Definition 4.5.

\subsubsection{Definitions on EC's Shell}

The shell of EC gives the scale of neighborhood of all emotional elements that is uncertainty called "soft membrane" which is defined by the following.

Definition 4.7. Upper approximation shell: $U P_{B}=\left\{P_{l} \mid P_{l} \in N_{P_{K}}^{\varepsilon_{u}}\right\}$, lower approximation Shell: $L P_{B}=\left\{P_{l} \mid P_{l} \in N_{P_{K}}^{\varepsilon_{l}}\right\}$, then shell of EC is $P_{B}=U P_{B} \backslash L P_{B}$, which denoted by $N(u, v)$, where $u, v$ are related to the density function. 


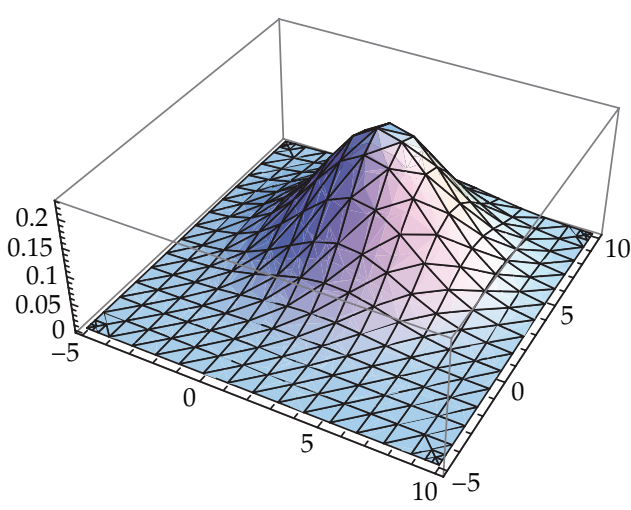

(a)

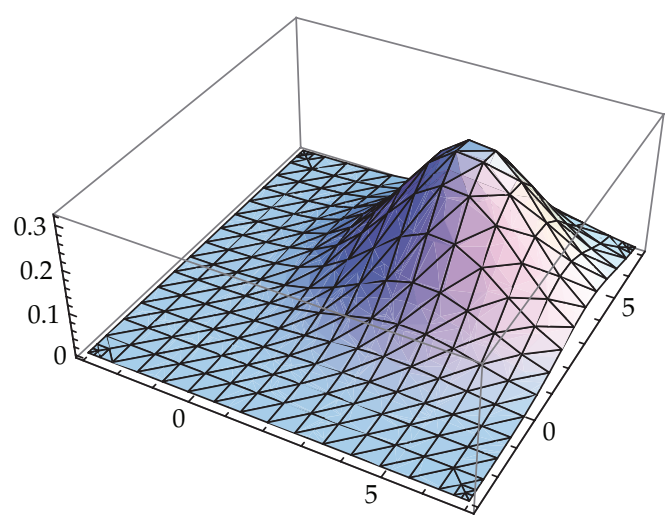

(b)

Figure 3: Different parameters in EC models also are subject to density function requirements. (a) Different $c$ and $\sigma$ that are subjected to formula as follows: $\rho_{(v, a)}=(1 /$ $\left.\sqrt{2 \pi \sigma_{1} \sigma_{2}} \sqrt{\left(1-r^{2}\right)}\right) e^{(-1 / 2)\left[\left(v-c_{1}\right)^{2}-2 r\left(v-c_{1}\right)\left(a-c_{2}\right)+\left(a-c_{2}\right)^{2} /\left(\sigma_{1}^{2}+\sigma_{2}{ }^{2}\right)\right]}=\left(1 / \sqrt{2 \pi \sigma_{1} \sigma_{2}}\right) e^{(-1 / 2)\left(\left(v-c_{1}\right)^{2}+\left(a-c_{2}\right)^{2} /\left(\sigma_{1}^{2}+\sigma_{2}{ }^{2}\right)\right)}(r=$ $0)$. (b) The same $c$ and $\sigma$ that referred to $\theta=(3.69,3.69,1.23 I)$.

\subsubsection{Probability Density of EC Employing Gaussian Model}

For singular- and circular-type kernel of EC, single Gaussian model (SGM) was applied to describe the density of these points, defined by

$$
\rho(P ; \mu, \Delta)=\frac{1}{\sqrt{(2 \pi)^{3}}|\Delta|} e^{-(1 / 2)(P-\mu)^{T} \Delta^{-1}(P-\mu)},
$$

where $\Delta$ is covariance matrix, $\mu$ is the central point of density function, the density function's properties are inducted by $(\Delta, \mu)$, so, this is a parameter estimation problem (shown in Figure 3).

And for plat-type kernel of EC, multi-SGMs will be addressed to resolve this problem,

$$
G(P)=\sum_{i} \alpha_{i} \rho\left(P ; \mu, \Delta_{i}\right), \quad i=1,2, \ldots n,
$$

where $\sum_{i} \alpha_{i}=1, G(P)$ is a Gaussian mixture model (GMM) (shown in Figure 4).

\subsection{FCBR of Product Style}

Firstly, we need to define the target problems, while using EC, we have the following.

Definition 4.8. Given a universe $\Omega=\left\{Q_{i}: i=1,2, \ldots, n\right\}$, it is a finite set, let $Q_{i} \in \Omega$ be a case of case space which is formalized as a triple: $Q_{i}=\left(P_{i}, \rho\left(P_{i}\right), G\left(P_{i}\right)\right)$, where $P_{i}$ is the kernel of EC, $\rho\left(P_{i}\right)$ is the probability density function of $P_{i}$ that is used to set the weight of cases, and $G\left(P_{i}\right)$ is a EC which contains $P_{i}$ as its kernel. 


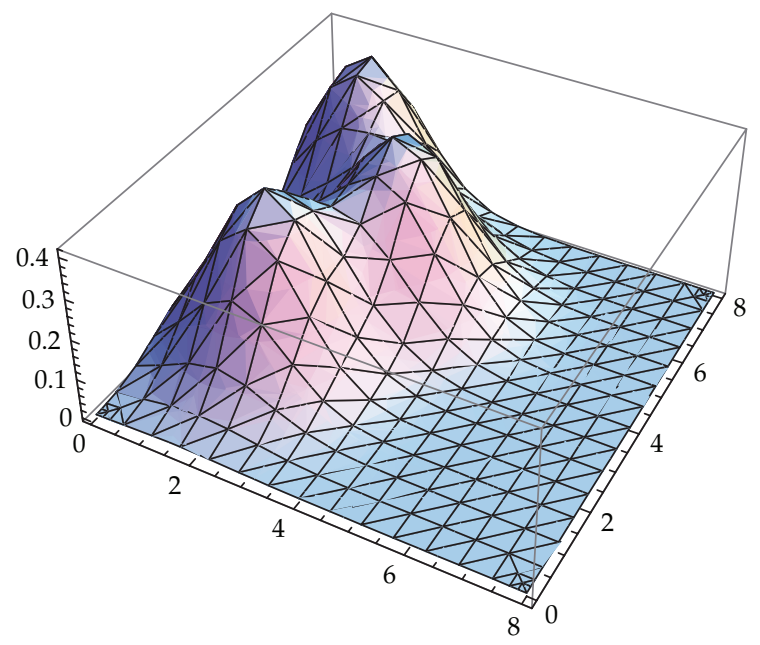

Figure 4: Overlapping appearance of different type kernel of EC, $\rho(P)=(2 \pi)^{-(1 / 2)}\left(e^{-(1 / 2)\left((x-1.71)^{2}+(y-2.79)^{2}\right)}+\right.$ $\left.e^{-(1 / 2)\left((x-3.05)^{2}+(y-5.25)^{2}\right)}+e^{-(1 / 2)\left((x-1.05)^{2}+(y-7.25)^{2}\right)}\right)$.

Definition 4.9. Given a product features space $\Phi=\left\{C_{i}: i=1,2, \ldots, m\right\}$, for each product $C_{i}=\left\{F_{i 1}, F_{i 2}, \ldots, F_{i k}\right\} \in \Phi$, it is a finite set, where $F_{i}$ is form feature. Let $\operatorname{Style}\left(C_{i}\right) \subset C_{i}$ be style features subset of $C_{i}$ that is the features relative to product style in $F_{i}$.

Definition 4.10. For any two style feature subset Style $\left(C_{K}\right)$ and $\operatorname{Style}^{\prime}\left(C_{K}\right) \subset C_{K}$, we defined that

(1) Style $\left(\neg C_{K}\right)=\operatorname{Style}\left(C_{K}\right)^{c}$,

(2) Style $\left(C_{K}\right) \backslash$ Style $^{\prime}\left(C_{K}\right)=\operatorname{Style}\left(C_{K}\right) \cap$ Style $^{\prime}\left(C_{K}\right)^{c}$.

Definition 4.11. Let Style $\left(C_{K}\right)^{*}$ be the extend set of Style $\left(C_{K}\right)$ Style $\left(C_{K}\right) \subset \operatorname{Style}\left(C_{K}\right)^{*}$, which denotes the new style features set after revise.

Construct a mapping between product feature space and case space-based EC: $\Phi \rightarrow$ $\Omega:\left\{\operatorname{Style}\left(C_{i}\right) \mapsto Q_{i}\right\}$, that is $Q_{i}=\left(P_{i}, \rho\left(P_{i}\right), G\left(P_{i}\right)\right)$ is applied to describe Style $\left(C_{i}\right)$.

Definition 4.12. For case base $C=\left\{C_{i}: i=1,2, \ldots, k\right\}$ and $C_{i}=\left\{F_{i 1}, F_{i 2}, \ldots, F_{i k}\right\} \in \Phi$, mapping $F \rightarrow Q$, we have that

$$
F_{i j}=\left\{v_{i j}, a_{i j}\right\}
$$

where $v_{i j}$ denotes valence scale relative to form and $a_{i j}$ relative to arousal. That is said form feature is mapped into a point in valence-arousal space.

Definition 4.13. The distance between form feature $F_{i j}$ and EC-Q is defined as Euclidian distance in valence-arousal space under Definition 4.2.

Definition 4.14. let Style $\left(C_{K}\right)>\operatorname{Style}^{1}\left(C_{K}\right)>\operatorname{Style}^{2}\left(C_{K}\right) \cdots>\operatorname{Style}^{*}\left(C_{K}\right)$ be a strictly increasing nested set, Style $\left(C_{K}\right)$ be a stable style feature set while studied from CBR process. 
Definition 4.15 (FNN). Let case base $C$ be involved of EC $Q=G(P)$, the similarity between the new case $C_{i}=\left\{F_{i 1}, F_{i 2}, \ldots, F_{i l}\right\}$ and previous case was defined by

$$
\operatorname{Sim}\left(C, C_{i}\right)=\sum_{j=1}^{l} d\left(F_{i j}, G(P)\right) \cdot \rho\left(F_{i j}\right)
$$

where $d\left(F_{i j}, G(P)\right)=\inf \left\{d\left(F_{i j}, Y_{l}\right): \forall Y_{l} \in G(P)\right\}, \rho\left(F_{i j}\right)$ is probability density function of $F_{i j}$ in valence-arousal space.

Definition 4.16. Style features subset calculation under fuzzy expression for new case:

$$
C_{i}=\left\{F_{i 1}, F_{i 2}, \ldots, F_{i l}\right\}=\left\{\frac{F_{i 1}}{\rho\left(F_{i 1}\right)}+\frac{F_{i 2}}{\rho\left(F_{i 2}\right)}+\cdots+\frac{F_{i l}}{\rho\left(F_{i l}\right)}\right\}
$$

where, $\rho\left(F_{i j}\right)$ is the membership of $F_{i j}$ belonging to EC, the similarity is calculated by Definition 4.15. Style features from new case were calculated by

$$
\text { Style }\left(C_{i}\right)=\left\{\sum_{j} F_{i j}: d\left(F_{i j}, G(P)\right) \cdot \rho\left(F_{i j}\right)<\delta\right\},
$$

where, $\delta=(1 / n m) \sum_{i} \sum_{j} d\left(F_{i j}, G(P)\right) \cdot \rho\left(F_{i j}\right), n$ is the total number of case base, $m$ is total number of features, that is, if a feature of new cases is less than the average fuzzy nearest neighbor of previous case, then it can be added in case base. By the above equation, style feature subset comes from all the features whose membership value are less than a given threshold, and this threshold value will affect the accuracy of case studies.

Definition 4.17. For new case $C_{i}$, if its fuzzy membership is less than a given threshold $\delta$ by Definition 4.16, then let $C^{*}=C \cup C_{i}$, while Style $(C)^{*}$ is the extend set of Style $(C)$ that is $C_{i}$ is retained.

The frame works of FCBR process was illustrated by Figure 5 .

\section{Case Study}

\subsection{Construction of Product Database}

The mobile phone was classified under form, layout (geometric connection), color, and material. In form feature classification, there have been 8 subclassifications: let $\{A, B, C, D, E, F, H\}=\{$ overall body form, ratio of body's length and width, body's thickness, shape of the screen cover, bottom form, body's edges line, proportion between screen and body, form of the number keys\} listed in Table 2.

In layout classification, there have been 6 sub-classifications, let $(I, J, K, L, M, N)=$ arrangement of the numeric keys, function keys layout, the fuselage edge treatment, the number of the basic components, the basic operation way, surface segmentation approach which is listed in Table 3. 


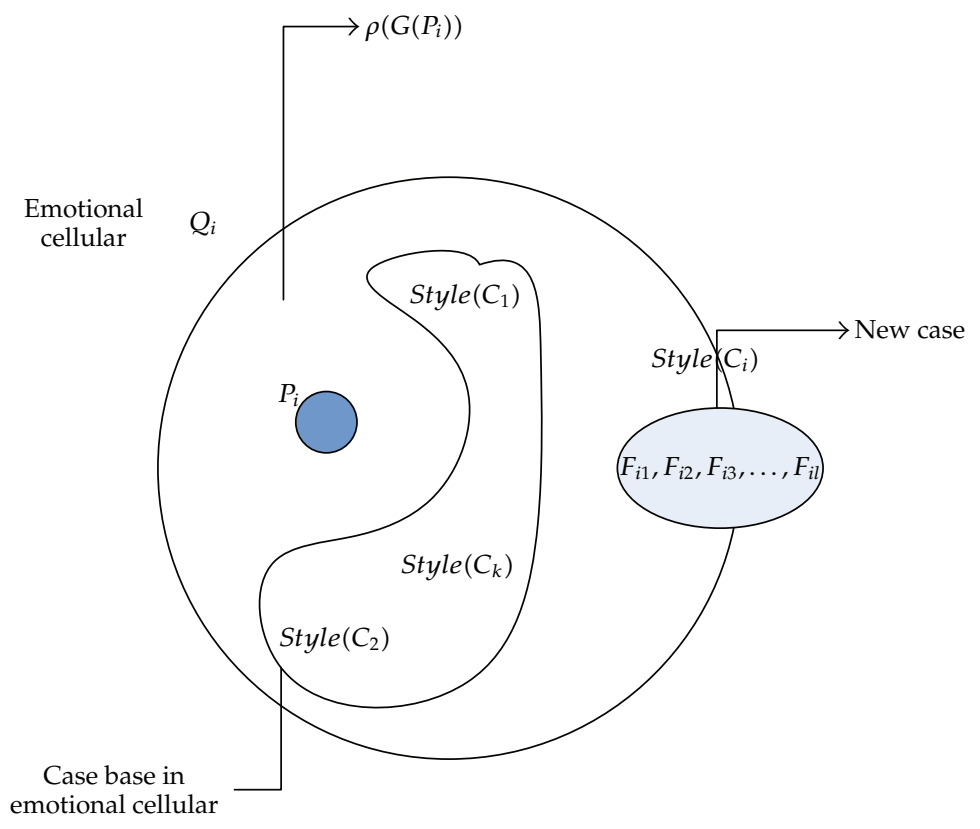

Figure 5: Similarity computing for new case in valence-arousal emotional space.

Table 2: Form classification and feature coding.

\begin{tabular}{|c|c|}
\hline Class & Form features \\
\hline $\bar{A}$ & $\{A 1$. linear, $A 2$. convex arc, $A 3$. concave arc, $A 4$. prominence waist $\}$ \\
\hline$B$ & $\{B 1.1: 1 \sim 1.5, B 2.1: 1.5 \sim 2, B 3.1: 2 \sim 2.5\}$ \\
\hline$C$ & $\{C 1$. thinner, $C 2$. thin, $C 3$. thick, $C 4$. thicker $\}$ \\
\hline$D$ & $\begin{array}{l}\text { \{D1. independent to function keys, } D 2 \text {. combination with function key, } D 3 \text {. partly overlap with } \\
\text { function keys\} }\end{array}$ \\
\hline$E$ & $\{$ E1. small $\mathrm{R}$ angle arc, E2. arcs, E3. arc-shaped $\}$ \\
\hline$F$ & $\{F 1$. straight, F2. Small arc, F3. big arc $\}$ \\
\hline G & $\{G 1.1: 1, G 2.1: 1 \sim 2, G 3.1: 2 \sim 3$, G4. other (large screen) $\}$ \\
\hline$H$ & $\begin{array}{l}\text { \{H1. rectangular, } H 2 \text {. oval shaped, } H 3 \text {. round, } H 4 \text {. other geometric shape, } H 5 \text {. divided by the } \\
\text { surface shape\} }\end{array}$ \\
\hline
\end{tabular}

Table 3: Geometric connections and feature coding.

\begin{tabular}{|c|c|}
\hline Class & Form features \\
\hline I & $\begin{array}{l}\text { \{11. same forms arranged in neat rows, I2. same forms but changeable alignment, I3. form } \\
\text { changeable but in neat rows, I4. form changeable and changeable alignment }\}\end{array}$ \\
\hline$J$ & $\{J 1$. large function keys, J2. symmetric dispersion function keys $\}$ \\
\hline K & $\{K 1$. near to $0, K 2$. small $R$ angle, $K 3$. middle $\mathrm{R}$ angle, $K 4$. big $\mathrm{R}$ angle, $K 5$. semirounded $\}$ \\
\hline$L$ & $\{L 1$. two, $L 2$. three, $L 3$. four, $L 4$. five, $L 5$. six and more $\}$ \\
\hline$M$ & $\{$ M1. flip, $M 2$. slide, $M 3$. straight, $M 4$. rotation $\}$ \\
\hline$N$ & $\{N 1$. by cover, $N 2$. by frame, $N 3$. by screen $\}$ \\
\hline
\end{tabular}


Table 4: Color and materials and their codes.

\begin{tabular}{|c|c|}
\hline Class & Form features \\
\hline $\mathrm{O}$ & $\{$ O1. metal decorative box, O2. printed decorative box, O3. gradient, $\mathrm{O} 4$. groove carving, $\mathrm{O}$. none $\}$ \\
\hline$P$ & $\{P 1$. silver, $P 2$. black, $P 3$. other $\}$ \\
\hline$Q$ & $\begin{array}{l}\text { \{Q1. silver blue, } Q 2 \text {. silver rose, } Q 3 \text {. black, } Q 4 \text {. swamp ash, } Q 5 \text {. light blue, } Q 6 \text {. pink, } Q 7 \text {. milk } \\
\text { white, } Q 8 \text {. silver, } Q 9 \text {. deep blue of venice, } Q 10 \text {. deep in paris gold, } Q 11 \text {. deep red rome\} }\end{array}$ \\
\hline$R$ & $\begin{array}{l}\{R 1 \text {. painting, } R 2 \text {. bright side, } R 3 \text {. acrylic, } R 4 \text {. metal, } R 5 \text {. plastic + rubber (Silicon), } R 6 \text {. key-film } \\
\text { (IMD), } R 7 \text {. other }\}\end{array}$ \\
\hline
\end{tabular}

Table 5: 2-dimensional color style evaluation ("Y"-selected).

\begin{tabular}{|c|c|c|c|c|c|c|c|}
\hline Words & & $\begin{array}{c}\text { A } \\
\text { Fortunately }\end{array}$ & $\begin{array}{c}\text { B } \\
\text { Active }\end{array}$ & $\begin{array}{c}\mathrm{C} \\
\text { Varied }\end{array}$ & $\begin{array}{c}\text { D } \\
\text { Lucky }\end{array}$ & $\begin{array}{c}\mathrm{E} \\
\text { Interesting } \\
\end{array}$ & $\begin{array}{c}\text { F } \\
\text { Peaceful }\end{array}$ \\
\hline 1 & Optimistic & & & & & & \\
\hline 2 & Stimulation & & & & Y & & \\
\hline 3 & Sexy & & Y & & & & \\
\hline 4 & Intense & & & & & Y & \\
\hline 5 & Aggression & & & & Y & & \\
\hline 6 & Gorgeous & & & & & & \\
\hline 7 & Lively & & & & Y & & \\
\hline 8 & Elegant & & Y & & & & \\
\hline 9 & Rich & & & & Y & & \\
\hline 10 & Mature & & & & & & \\
\hline 11 & Healing & & & Y & & & \\
\hline 12 & Wisdom & & & & & & \\
\hline 13 & Cool & & & & & & \\
\hline 14 & Protection & & & & & & \\
\hline 15 & Security & & & Y & & & \\
\hline 16 & Loyalty & & & & Y & & \\
\hline
\end{tabular}

In color and materials classification, there have been four subclasses: let $(O, P, Q, R)=$ \{screen edge processing, the keyboard color, body color, body surface treatment\}, Table 4 shows the specific subclass and their coding.

In color evaluation, 2-dimensional scale was applied in system shown in Table 5.

\subsection{Case Construction and Reasoning}

Let $\mathrm{F}=[A, B, C, D, E, F, G, H]$ be form matrix, the values are relative coding of each selection, for example, $F=[1,3,2,3,3,3,4,5]$ denotes $[A 1, B 3, C 2, D 3, E 3, F 3, G 4, H 5]$, and let $G=[I, J$, $K, l, M, N], C=[O, P, Q, R]$, under fuzzy similarity computing was under definitions in Section 4.2.

\subsection{Result and Discussion}

78 mobile phones were used to construct case base through online questionnaire system. First, these 78 Nokia mobile phones will be formalized as $(F, G, C)$, and whether those cases' features belong to product style or not depends on the result from FCBR under the proposed 
Table 6: Style description, representative product and kernel features codes (Nokia).

\begin{tabular}{lccc}
\hline Style & Product & EC characterize code & Color Evaluation \\
\hline Classical & $\mathrm{N} 93$ & C3E211M1 & $6 \mathrm{D}$ \\
Aristocratic & 8800 & E3G1M2R4Q2 & $7 \mathrm{D}$ \\
Rock & $\mathrm{N} 70$ & A1B2M3 & $10 E$ \\
Dynamic Black & $\mathrm{N} 81$ & B3E1G4Q3R6 & $12 \mathrm{~F}$ \\
Adore & 7088 & A3E2G2K4Q7 & $9 \mathrm{~A}$ \\
Metal & 6275 & E211M2Q8 & $12 \mathrm{~B}$ \\
Classic Nordic & $\mathrm{E} 50$ & B3C1D2M3 & $1 B$ \\
Bohemian & 7373 & G3Q6 & $3 F$ \\
Luxury & 7500 & D2H5J2Q3 & $2 F 6 A$ \\
Apple iPod & N73 & G113M2Q7 & $6 A$ \\
Simple business & E51 & I3O3P2R7 & $3 E$ \\
Modern fashion & 7900 & D2H4P2 & $3 E$ \\
Sport Transformation & 5300 & H2M2 & $4 \mathrm{~B} 6 \mathrm{D}$ \\
\hline
\end{tabular}

similarity definitions mentioned above; for the same case in questionnaire system, it can seem to be the revise operation. If the similarity between new case and average case is less than a given threshold, then this new case will be retained. Partly cases were reserved to reflect the product style, and the critical form features were listed in Table 6. For example, "classic nordic" E50, its encoding is [A1B3C1D2E1F1G3H1, I1J2K1L4M3N, 3O4P3Q4R4]. Evaluation by two-dimensional color system is "optimistic" and "Active." By FCBR process, average case is [B3C1D2M3]; with the style characteristics of the mobile phone has a metal body panels, T-shaped key generally placed in the phone right way, navigation key with control rods, digital array key layout, color friendly and simple, calm, and so forth. The results indicated that all features listed in Table 6 belong to Nokia traditional style which meet the high-ended business application need in market position.

\section{Discussing and Concluding Remarks}

\subsection{Comparing with Traditional Methods on Product Style Evaluation}

Comparing with traditional methods, such as KANSEI engineering, it applied KANSEI adjectives on perceptual evaluation and also needed the appropriate degree of adverbs to indicate customers' preference on every product characteristics, such as "very" and "extreme". However, this scale has great randomness and cannot be directly used for calculation; further improvement is Likert scale that used psychology to reflect the preference. It used scores to distinguish emotional level such as 5-point scale and 7-point scale; scores also can distinguish the corresponding positive or responsible for the emotional level, but the scale method is far too simple. The use of semantic differential method still does not solve the complex emotional quantitative problem, and also cannot reflect the perception of ambiguity. In this respect, fuzzy set was considered to be an effective solution. In the fuzzy set theory, the user is represented as a composite perceptual general form of fuzzy sets, and with the logical calculation on fuzzy set, the user's overall emotional evaluation on products' implicit emotion would be extracted, the methodology combining genetic algorithm was adopted in our works on TV style research [40]. Therefore, the linear function on weights distribution 
seems that it cannot reflect the exact expression of human's emotion on product style. So, EC will be more feasible to deal with this problem. It also illustrated the effectiveness on case study of this paper.

\subsection{Conclusions and Further Research}

The form, color, and materials of product played a decisive role in the formation of product style by researching expression and reasoning of style knowledge; due to the uncertainty of product style, FCBR was addressed to resolve this problem and product style was formalized as an EC, the similarity between cases was also defined from EC's probability density function, in the case study, this model was proved to be effective, but, it had also the following problems.

(1) Similarity computing in the style of the weight of morphological characteristics' calculation is too complex while depend on probability density function of EC that limit the size of the experiment, and the accuracy of this density function remains to be further studied.

(2) Besides the form, layout, color, and materials, there are other factors, such as the use of functional brand recognition product emotional experience will also influence the product style formation. Therefore, the extraction of knowledge of product style was improved and developed continuously.

In the next step, case base will be established in a large scale while similarity computing must be under more reasonable definitions, weight distribution program, and inference system also would be improved, that is to carry out the depth mining for product style.

\section{Acknowledgments}

This paper is funded by Natural Science Foundation of Zhejiang Province under Grant no. Y1110322 and National Natural Science Foundation under Grant no. 61070075.

\section{References}

[1] J. A. Russell, “A circumplex model of affect,” Journal of Personality and Social Psychology, vol. 39, no. 6, pp. 1161-1178, 1980.

[2] M. M. Bradley, M. K. Greenwald, M. C. Petry, and P. J. Lang, "Remembering pictures: pleasure and arousal in memory," Journal of Experimental Psychology, vol. 18, no. 2, pp. 379-390, 1992.

[3] C. Stickel, M. Ebner, S. Steinbach-Nordmann, G. Searle, and A. Holzinger, "Emotion detection: application of the valence arousal space for rapid biological usability testing to enhance universal access," in Proceedings of the 5th International Conference on Universal Access in Human-Computer Interaction (UAHCI '09), C. Stephanidis, Ed., vol. 5614 of Lecture Notes in Computer Science, pp. 615-624, Springer, 2009.

[4] G. Chanel, J. Kronegg, D. Grandjean, and T. Pun, "Emotion assessment: arousal evaluation using EEG's and peripheral physiological signals," in Proceedings of the Workshop on Multimedia Content Representation, Classification and Security (MRCS '06), B. Gunsel et al., Ed., vol. 4105 of Lecture Notes in Computer Science, pp. 530-537, Springer, Berlin, Germany, 2006.

[5] J. León-Carrión, J. F. Martín-Rodríguez, J. Damas-López et al., “A lasting post-stimulus activation on dorsolateral prefrontal cortex is produced when processing valence and arousal in visual affective stimuli," Neuroscience Letters, vol. 422, no. 3, pp. 147-152, 2007. 
[6] P. A. Lewis, H. D. Critchley, P. Rotshtein, and R. J. Dolan, "Neural correlates of processing valence and arousal in affective words," Cerebral Cortex, vol. 17, no. 3, pp. 742-748, 2007.

[7] J. Lawry and Y. Tang, "Uncertainty modelling for vague concepts: a prototype theory approach," Artificial Intelligence, vol. 173, no. 18, pp. 1539-1558, 2009.

[8] Y. Tang and J. Lawry, "Linguistic modelling and information coarsening based on prototype theory and label semantics," International Journal of Approximate Reasoning, vol. 50, no. 8, pp. 1177-1198, 2009.

[9] F. Shi, Employing valence-arousal on representation and reasoning of product's implicit emotion, Ph.D. thesis, Zhejiang University, China, Zhejiang, China, 2011.

[10] P. Perner, "Case-based reasoning and the statistical challenges," in Proceedings of the 9th European conference on Advances in Case-Based Reasoning (ECCBR '08), K.-D. Althoff et al., Ed., vol. 5239 of Lecture Notes in Artificial Intelligence, pp. 430-443, Springer, Berlin, Germany, 2008.

[11] D. Patterson, N. Rooney, M. Galushka, V. Dobrynin, and E. Smirnova, "SOPHIA-TCBR: a knowledge discovery framework for textual case-based reasoning," Knowledge-Based Systems, vol. 21, no. 5, pp. 404-414, 2008.

[12] M. Salamó and M. López-Sánchez, "Rough set based approaches to feature selection for Case-Based Reasoning classifiers," Pattern Recognition Letters, vol. 32, no. 2, pp. 280-292, 2011.

[13] A. Khanum, M. Mufti, M. Y. Javed, and M. Z. Shafiq, “Fuzzy case-based reasoning for facial expression recognition," Fuzzy Sets and Systems, vol. 160, no. 2, pp. 231-250, 2009.

[14] S. T. Li and H. F. Ho, "Predicting financial activity with evolutionary fuzzy case-based reasoning," Expert Systems with Applications, vol. 36, no. 1, pp. 411-422, 2009.

[15] Y. J. Jiang, J. Chen, and X. Y. Ruan, “Fuzzy similarity-based rough set method for case-based reasoning and its application in tool selection," International Journal of Machine Tools and Manufacture, vol. 46, no. 2, pp. 107-113, 2006.

[16] T. Y. Slonim and M. Schneider, "Design issues in fuzzy case-based reasoning," Fuzzy Sets and Systems, vol. 117, no. 2, pp. 251-267, 2001.

[17] P. C. Chang, C. Y. Fan, and W. Y. Dzan, "A CBR-based fuzzy decision tree approach for database classification," Expert Systems with Applications, vol. 37, no. 1, pp. 214-225, 2010.

[18] R. Schank, Dynamic Memory: A Theory of Learning in Computers and People, Cambridge University Press, New York, NY, USA, 1982.

[19] J. L. Kolodner, "Reconstructive memory: a computer model," Cognitive Science, vol. 7, no. 4, pp. 281328, 1983.

[20] M. Lebowitz, "Memory-based parsing," Artificial Intelligence, vol. 21, no. 4, pp. 363-404, 1983.

[21] E. Hüllermeier, I. Vladimirskiy, B. Prados Suarez et al., "Supporting case-based retrieval by similarity skylines: basic concepts and extensions," in Proceedings of the 9th European conference on Advances in Case-Based Reasoning (ECCBR '08), K.-D. Althoff et al., Ed., vol. 5239 of Lecture Notes in Artificial Intelligence, pp. 240-254, Springer, Berlin, Germany, 2008.

[22] M. C. Wu, Y. F. Lo, and S. H. Hsu, "A fuzzy CBR technique for generating product ideas," Expert Systems with Applications, vol. 34, no. 1, pp. 530-540, 2008.

[23] M. Y. Cheng, H. C. Tsai, and Y. H. Chiu, "Fuzzy case-based reasoning for coping with construction disputes," Expert Systems with Applications, vol. 36, no. 2, pp. 4106-4113, 2009.

[24] R. Mugge, P. C. M. Govers, and J. P. L. Schoormans, "The development and testing of a product personality scale," Design Studies, vol. 30, no. 3, pp. 287-302, 2009.

[25] H. Cai, W. He, and D. Zhang, "A semantic style driving method for products' appearance design," Journal of Materials Processing Technology, vol. 139, no. 1-3, pp. 233-236, 2003.

[26] S. H. Han, K. J. Kim, M. H. Yun, S. W. Hong, and J. Kim, "Identifying mobile phone design features critical to user satisfaction," Human Factors and Ergonomics In Manufacturing, vol. 14, no. 1, pp. 15-29, 2004.

[27] A. Aamodt and E. Plaza, “Case-based reasoning: foundational issues, methodological variations, and system approaches," Artificial Intelligence Communications, vol. 7, no. 1, pp. 39-59, 1994.

[28] I. Watson, "Case-based reasoning is a methodology not a technology," Knowledge-Based Systems, vol. 12, no. 5-6, pp. 303-308, 1999.

[29] Z. Shen, H. C. Lui, and L. Ding, "Approximate case-based reasoning on neural networks," International Journal of Approximate Reasoning, vol. 10, no. 1, pp. 75-98, 1994.

[30] D. W. Aha, "The omnipresence of case-based reasoning in science and application," Knowledge-Based Systems, vol. 11, no. 5-6, pp. 261-273, 1998.

[31] E. Fix and J. L. Hodge Jr., Discriminatory analysis, nonparametric discrimination, consistency properties. U.S. Air Force Sch. Aviation Medicine, Randolf Field, Texas, Project 21-49-004, Contract AF 41 (128)31, Rep. 4, 1951. 
[32] G. Glass, T. S. Bhatia, J. C. Hiebert et al., "The measurement of KNN and KLL in at $800 \mathrm{MeV,"} \mathrm{Physics}$ Letters B, vol. 129, no. 1-2, pp. 27-30, 1983.

[33] P. A. Devijver and J. Kittler, "Pattern recognition: a statistical approach," Pattern Recognition, 1982.

[34] D. L. Wilson, "Asymptotic properties of nearest neighbor rules using edited data," IEEE Transactions on Systems, Man and Cybernetics, vol. 2, no. 3, pp. 408-421, 1972.

[35] L. I. Kuncheva, "Fitness functions in editing k-NN reference set by genetic algorithms," Pattern Recognition, vol. 30, no. 6, pp. 1041-1049, 1997.

[36] Y. C. Liaw, M. L. Leou, and C. M. Wu, "Fast exact k nearest neighbors search using an orthogonal search tree," Pattern Recognition, vol. 43, no. 6, pp. 2351-2358, 2010.

[37] J. M. Keller, M. R. Gray, and J. A. Givens, "A fuzzy k-nearest neighbor algorithm," IEEE Transactions on Systems, Man and Cybernetics, vol. 15, no. 4, pp. 580-585, 1985.

[38] O. Mangasarian and G. Kou, "Feature selection for nonlinear kernel support vector machines," in Proceedings of the 7th IEEE International Conference on Data Mining (ICDM '07), pp. 231-236, Omaha, Neb, USA, October 2007.

[39] W. Wang, Z. Xu, W. Lu, and X. Zhang, "Determination of the spread parameter in the Gaussian kernel for classification and regression," Neurocomputing, vol. 55, no. 3-4, pp. 643-663, 2003.

[40] J. Xu, S. Sun, Z. Tan, and F. Shi, "An interactive evolutionary design system with feature extraction," in Proceedings of the 12th International Conference on Human-Computer Interaction (HCI '07), J. Jacko, Ed., vol. 4553 of Lecture Notes in Computer Science, pp. 1085-1094, Beijing, China, July 2007. 


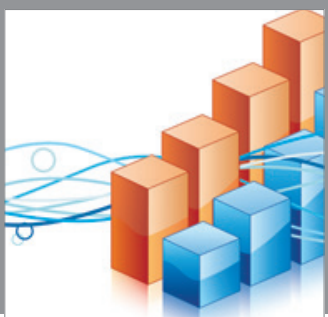

Advances in

Operations Research

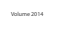

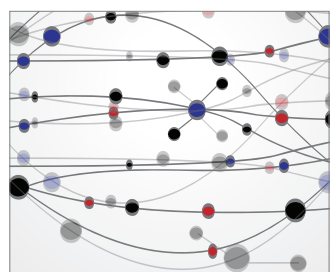

\section{The Scientific} World Journal
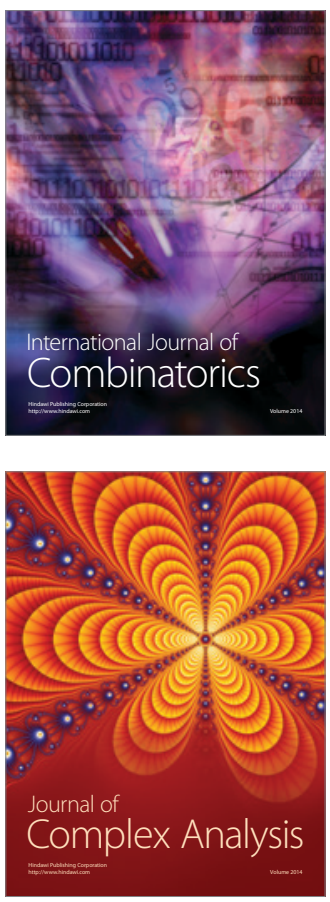

International Journal of

Mathematics and

Mathematical

Sciences
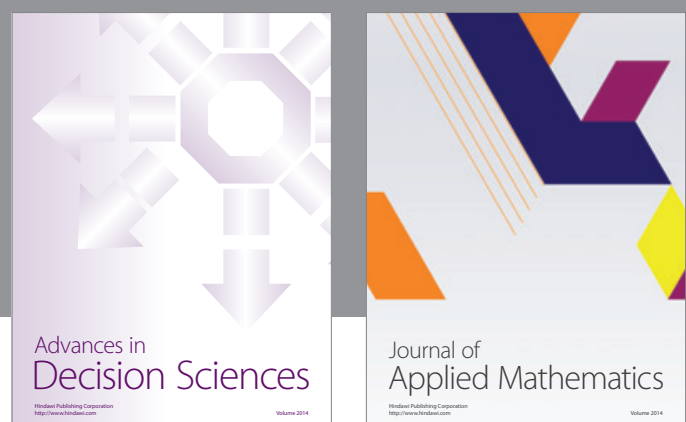

Journal of

Applied Mathematics
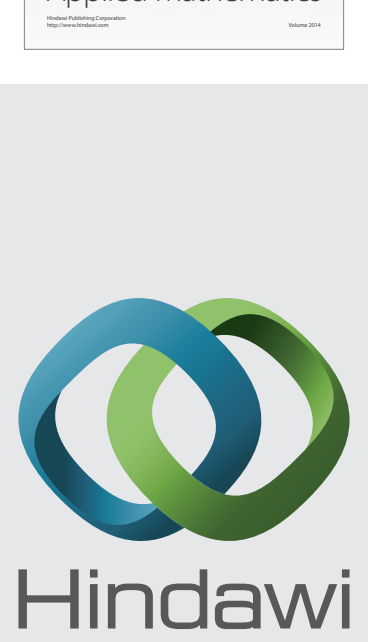

Submit your manuscripts at http://www.hindawi.com
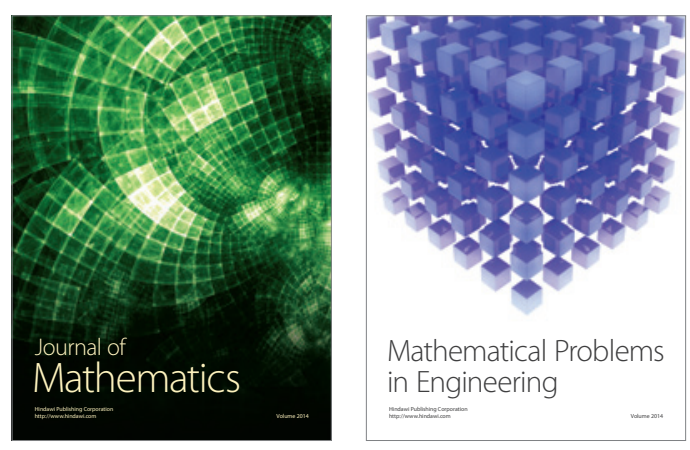

Mathematical Problems in Engineering
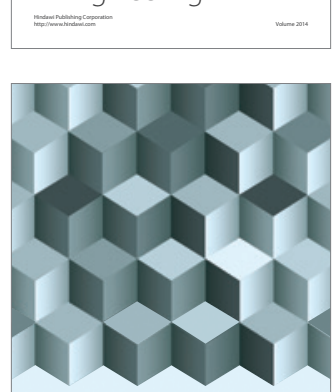

Journal of

Function Spaces
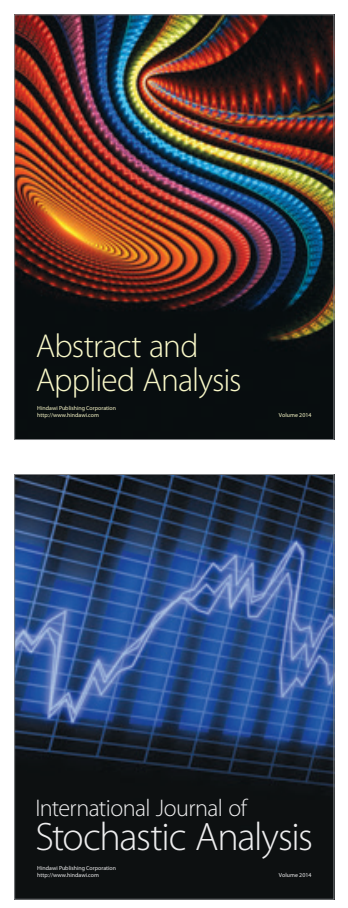

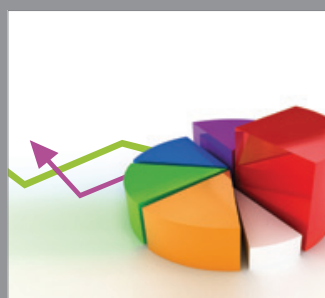

ournal of

Probability and Statistics

Promensencen
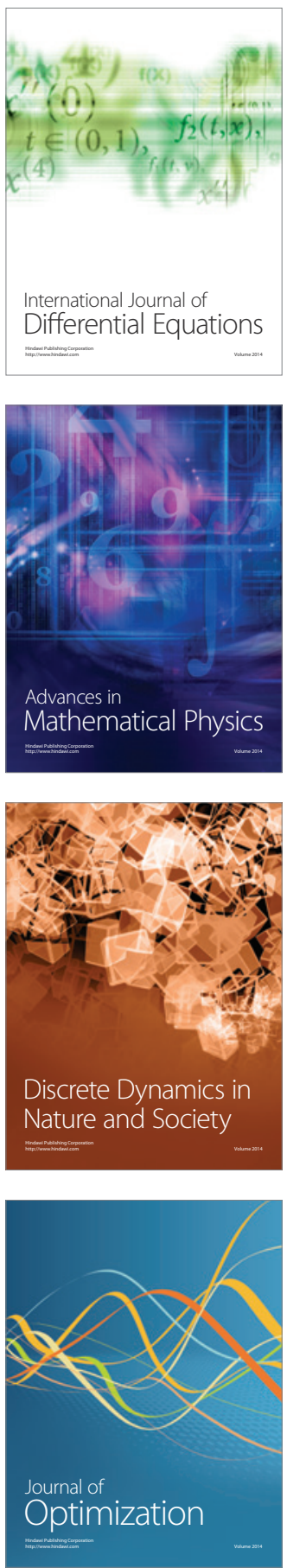